\title{
The Diagnostic Significance of Usual Biochemical Parameters in Coronavirus Disease 19 (COVID-19): Albumin to Globulin Ratio and CRP to Albumin Ratio
}

\author{
Gavriela M. Feketea ${ }^{1,2 *}$ and Vasiliki Vlacha ${ }^{3 *}$ \\ ${ }^{1}$ Department of Hematology, "uliu Hatieganu" University of Medicine and Pharmacy, Cluj-Napoca, Romania, ${ }^{2}$ Department of \\ Pediatrics, Pediatric Allergy Outpatient Clinic, Karamandaneio Children Hospital of Patras, Patras, Greece, ${ }^{3}$ Department of \\ Early Years Learning and Care, University of loannina, loannina, Greece
}

Keywords: albumin/globulin ratio (AGR), CRP/albumin ratio, COVID-19, SARS-CoV-2, kawasaki like disease, ARSD, ICU - intensive care unit

OPEN ACCESS

Edited by:

Ru-Ping Dai,

Central South University, China

Reviewed by:

Tuantuan Zhao,

Stanford University, United States

Bowen Wu,

Stanford University, United States

${ }^{*}$ Correspondence:

Gavriela M. Feketea

fechetea.gabriela@umfcluj.ro;

gabychri@otenet.gr

Vasiliki Vlacha

vasovlaha@gmail.com

Specialty section:

This article was submitted to

Intensive Care Medicine and

Anesthesiology,

a section of the journal

Frontiers in Medicine

Received: 28 May 2020 Accepted: 19 October 2020 Published: 03 November 2020

Citation: Feketea GM and Vlacha V (2020) The Diagnostic Significance of Usual

Biochemical Parameters in Coronavirus Disease 19 (COVID-19): Albumin to Globulin Ratio and CRP to

Albumin Ratio. Front. Med. 7:566591 doi: 10.3389/fmed.2020.566591
The clinical course of patients with COVID-19, and the progression to severe disease, are difficult to predict. There is a growing interest in identifying individuals who could be at greater risk for developing severe or critical COVID-19, acute respiratory distress syndrome (ARDS) in adults, Kawasaki-like disease in young individuals and children, and even death. Several laboratory parameters have been analyzed in conjunction with COVID-19. Specifically, significant elevation of C-reactive proteins, erythrocyte sedimentation rate, interleukin-6, and lactate dehydrogenase has been observed. On the other hand, the total white blood cell count and the eosinophilic and lymphocytic count have been decreased in COVID-19 patients. In a recent meta-analysis, increased CRP, lymphopenia, and increased LDH were significantly associated with the severity of the disease (1). The levels of certain laboratory values that proved to be elevated in cytokine storm (ferritin, procalcitonin, and troponin) may not be available at most hospital laboratories or are mainly used for research purposes (IL-6) $(2,3)$. On the other hand, CRP, albumin, and globulin are readily available, shortly after admittance, and are often part of an admission workup, in general hospitals and particularly in intensive care units (ICU).

Albumin and globulin are two important components of serum proteins and have been proven to be involved in systemic inflammation. A low serum albumin reflects a poor nutritional status, liver and kidney dysfunction, and has been shown to be an independent predictor of poor survival in critically ill patients. Furthermore, similar results were found regarding MERS (4). Decreased albumin at admission has been an independent risk factor associated with unimprovement during follow-up in COVID-19 patients (5). On the other hand, an increased globulin level may reflect a chronic inflammatory response. Thus, the additive effect of both albumin and globulin would not only be a prognostic factor for potential COVID-19 complications during the course of the illness, but also an initial risk index of SARS-CoV-2 positive individuals. Wu et al. showed recently that the level of albumin is significantly lower [ $30.40 \mathrm{~g} / \mathrm{L}(27.15-33.35)$ vs. $33.70 \mathrm{~g} / \mathrm{L}(30.95-36.30), p<$ $0.001]$ and the globulin level higher [31.60 g/L (29.35-35.05) vs. $30.00 \mathrm{~g} / \mathrm{L}(28.25-32.55), p=0.004]$ in COVID-19 patients with ARDS comparative with those without ARDS (6).

The albumin to globulin ratio (AGR) is calculated according to the following formula: AGR $=$ albumin/globulin. In young children, who to date are not at a high risk for developing critical COVID-19, the AGR is higher than in adults due to constitutionally lower globulin. Contrary to this, in hypertension, diabetes, cardiovascular disease, and cancer, which frequently are comorbidities in critical COVID-19, the AGR is lower than normal mainly due to 
hypoalbuminemia. In different solid tumor patients, a high AGR has a significant positive prognostic effect on survival (7). In patients hospitalized with $\mathrm{H}_{1} \mathrm{~N}_{1}$ infection, AGR reversal (ratio < 1) was associated with prolonged hospital stay, ICU admission, and ventilator use (8).

Two months after the spread of SARS-CoV-2 in Italy, Verdoni et al. found a 30-fold increased incidence of Kawasaki-like disease in children (9), a rare acute hyper-inflammatory syndrome emerging during the COVID-19 pandemic (10) with coronary artery aneurysms as its main complication. The potential for missed or late diagnosis and consequently delayed treatment of Kawasaki disease in children leading to an increased risk of coronary artery aneurysms, is of particular concern during this pandemic (11). Just before the pandemic, Mammadov et al. using a multivariate analysis found that a lower AGR served as an independent predictor of coronary artery aneurysms in children with Kawasaki disease and had a sensitivity of $56.25 \%$ and a specificity of $61.11 \%$ at a cutoff value of $<1.48$ (12).

Older age, chronic lung disease, cardiovascular disease, diabetes mellitus, obesity, immunocompromise, end-stage renal disease, and liver disease have been established as potential risk factors for severe COVID-19 (13). The AGR can be decreased in those conditions. In chronic kidney disease patients, it has been shown that AGR was associated with patient mortality of all causes as well as of cardiovascular diseases (14). Acute liver injury due to COVID-19 is associated with increased death risk, as it has been shown by Fu (15). The total protein, albumin, and albumin/globulin ratio were decreased in critically ill patients with acute liver injury compared with those with a less severe illness. In addition, the albumin levels were lower in patients with concurrent diabetes. In that analysis, it has been also shown that the fatality rate was higher in older patients and in ones with hypoproteinemia. A decreased albumin/globulin ratio has been also identified by Tian et al. as one of the risk factors related with the severity of COVID-19 in patients with cancer (16).

Taking all the above into consideration we believe that to calculate the AGR during a prolonged fever, at admission and also throughout the course of the illness, would be useful as a prognostic index for severe COVID-19 including ARDS and in young individuals and children with Kawasaki-like disease.

$\mathrm{C}$-reactive protein (CRP) induction is part of the acute phase response, in which the synthesis of many plasma proteins is increased, whereas a smaller number, particularly albumin, is decreased (17). It has been reported that the patients who needed invasive ventilation had increased inflammatory markers, including CRP (18). Hypoalbuminemia (5) and elevated CRP (19) has been shown to be associated with severe illness and death in patients with COVID-19. The CRP to albumin ratio has been shown to be more accurate than CRP alone for predicting the 28day mortality in critically ill patients (20). In addition, a higher CRP to albumin ratio at admission has been determined as a risk factor for in-hospital mortality in elderly patients with acute kidney injury requiring dialysis (21). Similarly, an initial elevated
CRP to albumin ratio was significantly related to 28 -day mortality associated with infections caused by Elizabethkingia spp., a dangerous opportunistic bacterial pathogen causing different illnesses including pneumonia (22). The authors concluded that the prediction of clinical courses using an initial CRP to albumin ratio is a priority to reduce the mortality in these patients. We have calculated the CRP to albumin ratio from the published data of an earlier publication by Liu et al. regarding SARS-CoV-2 infected patients (23). The median (range) of the ratio was 9.6 (1.0-2.5). One patient, who was diagnosed with shock and respiratory failure, had the highest value. It is also interesting to note that the patients with the lowest values were younger and without comorbidities. However, the study was limited to 12 patients.

Xie et al. found that CRP levels had a sensitivity of $65.52 \%$ and a specificity of $62.7 \%$ for predicting IVIG-resistance at a cutoff point of $>100 \mathrm{mg} / \mathrm{L}$ in children with Kawasaki disease. In addition, albumin $<32 \mathrm{~g} / \mathrm{L}$, had a sensitivity and specificity for predicting IVIG-resistance 72 and $83.19 \%$, respectively (24).

The three laboratory values, albumin, globulin, and CRP have been separately reported to be associated with normal aging. Healthy older people have been shown to have low serum levels of CRP and pro-inflammatory cytokines such as IL-6 compared with older people with comorbidities. However, those values are higher in aging people than in the younger population (25). Additionally, the increased levels of those parameters are associated with the raised risk of morbidity and mortality in the older subjects (26).

The albumin serum concentration has been shown to decrease with age (27-30). It has been also demonstrated that the globulin levels are higher in older people compared with younger people (31). Both nutritional and chronic inflammatory status, as it results from different comorbidities and aging, clearly influence the prognostic of COVID-19. In addition, inflammation due to COVID infection is added to this status.

There is an increased urgent need to detect new biomarkers in order to identify cases of COVID-19 that will evolve unfavorably in adults and children. These biomarkers must be easy to measure and accessible to most hospitals that manage COVID-19 cases. The proposed ratios (albumin to globulin and CRP to albumin) seem to be more accurate than each value separately and could be included in the initial assessment of patients that have tested PCR positive for SARS-CoV-2, in order to identify those who are at risk of developing ARDS or Kawasaki disease in young individuals and children. In addition, they can be measured during hospital or ICU admissions to evaluate the course of the illness.

\section{AUTHOR CONTRIBUTIONS}

GF and VV contributed to the design and implementation of the research, to the analysis of the results, and to the writing of the manuscript. All authors contributed to the article and approved the submitted version. 


\section{REFERENCES}

1. Zhang Z-L, Hou Y-L, Li D-T, Li F-Z. Laboratory findings of COVID-19: a systematic review and meta-analysis. Scand J Clin Lab Invest. (2020) 80:4417. doi: 10.1080/00365513.2020.1768587

2. Huang C, Wang Y, Li X, Ren L, Zhao J, Hu Y, et al. Clinical features of patients infected with 2019 novel coronavirus in Wuhan, China. Lancet. (2020) 395:497-506. doi: 10.1016/S0140-6736(20)30183-5

3. Coomes EA, Haghbayan H. Interleukin-6 in COVID-19: a systematic review and meta-analysis. medRxiv [Preprint]. (2020). doi: 10.1101/2020.03.30.20048058

4. Ko J-H, Park GE, Lee JY, Lee JY, Cho SY, Ha YE, et al. Predictive factors for pneumonia development and progression to respiratory failure in MERS-CoV infected patients. J Infect. (2016) 73:468-75. doi: 10.1016/j.jinf.2016.08.005

5. Zhang J, Wang X, Jia X, Li J, Hu K, Chen G, et al. Risk factors for disease severity, unimprovement, and mortality of COVID-19 patients in Wuhan, China. Clin Microbiol Infect. (2020) 26:767-72. doi: 10.1016/j.cmi.2020.04.012

6. Wu C, Chen X, Cai Y, Xia J, Zhou X, Xu S, et al. Risk factors associated with acute respiratory distress syndrome and death in patients with coronavirus disease 2019 pneumonia in Wuhan, China. JAMA Intern Med. (2020) 180:934-43. doi: 10.1001/jamainternmed.2020.0994

7. He J, Pan H, Liang W, Xiao D, Chen X, Guo M, et al. Prognostic effect of albumin-to-globulin ratio in patients with solid tumors: a systematic review and meta-analysis. J Cancer. (2017) 8:4002-10. doi: 10.7150/jca.21141

8. Ramaraju K, Murthy AK, Balasubramaniam N, Leon VK. Serum albuminglobulin ratio reversal predicts morbidity in patients hospitalized for influenza A(H1N1) infection. Eur Resp J. (2018) 52(Suppl. 62):PA2612. doi: 10.1183/13993003.congress-2018.PA2612

9. Verdoni L, Mazza A, Gervasoni A, Martelli L, Ruggeri M, Ciuffreda M, et al. An outbreak of severe Kawasaki-like disease at the Italian epicentre of the SARS-CoV-2 epidemic: an observational cohort study. Lancet. (2020) 395:1771-8. doi: 10.1016/S0140-6736(20)31103-X

10. Viner RM, Whittaker E. Kawasaki-like disease: emerging complication during the COVID-19 pandemic. Lancet. (2020) 395:1741-3. doi: 10.1016/S0140-6736(20)31129-6

11. Harahsheh AS, Dahdah N, Newburger JW, Portman MA, Piram M, Tulloh $\mathrm{R}$, et al. Missed or delayed diagnosis of Kawasaki disease during the 2019 novel coronavirus disease (COVID-19) pandemic. J Pediatr. (2020) 222:2612. doi: 10.1016/j.jpeds.2020.04.052

12. Mammadov G, Liu HH, Chen WX, Fan GZ, Li RX, Liu FF, et al. Hepatic dysfunction secondary to Kawasaki disease: characteristics, etiology and predictive role in coronary artery abnormalities. Clin Exp Med. (2020) 20:2130. doi: 10.1007/s10238-019-00596-1

13. Gandhi RT, Lynch JB, del Rio C. Mild or moderate Covid-19. N Engl J Med. (2020). doi: 10.1056/NEJMcp2009249. [Epub ahead of print].

14. Wu P-P, Hsieh Y-P, Kor C-T, Chiu P-F. Association between albumin-globulin ratio and mortality in patients with chronic kidney disease. J Clin Med. (2019) 8:1991. doi: 10.3390/jcm8111991

15. Fu L, Fei J, Xu S, Xiang H-X, Xiang Y, Tan Z-X, et al. Acute liver injury and its association with death risk of patients with COVID19: a hospital-based prospective case-cohort study. medRxiv [Preprint]. (2020). doi: 10.1101/2020.04.02.20050997

16. Tian J, Yuan X, Xiao J, Zhong Q, Yang C, Liu B, et al. Clinical characteristics and risk factors associated with COVID-19 disease severity in patients with cancer in Wuhan, China: a multicentre, retrospective, cohort study. Lancet Oncol. (2020) 21:893-903. doi: 10.1016/S1470-2045(20)30309-0

17. Black S, Kushner I, Samols D. C-reactive protein. J Biol Chem. (2004) 279:48487-90. doi: 10.1074/jbc.R400025200
18. Goyal P, Choi JJ, Pinheiro LC, Schenck EJ, Chen R, Jabri A, et al. Clinical characteristics of Covid-19 in New York City. N Engl J Med. (2020) 382:23724. doi: 10.1056/NEJMc2010419

19. Guan WJ, Ni ZY, Hu Y, Liang WH, Ou CQ, He JX, et al. Clinical characteristics of coronavirus disease 2019 in China. N Engl J Med. (2020) 382:170820. doi: 10.1056/NEJMoa2002032

20. Park JE, Chung KS, Song JH, Kim SY, Kim EY, Jung JY, et al. The C-reactive protein/albumin ratio as a predictor of mortality in critically Ill patients. J Clin Med. (2018) 7:333. doi: 10.3390/jcm7100333

21. Duarte I, Gameiro J, Resina C, Outerelo C. In-hospital mortality in elderly patients with acute kidney injury requiring dialysis: a cohort analysis. Int Urol Nephrol. (2020) 52:1117-24. doi: 10.1007/s11255-020-02482-2

22. Seong H, Kim JH, Kim JH, Lee WJ, Ahn JY, Choi JY, et al. Risk factors for mortality in patients with elizabethkingia infection and the clinical impact of the antimicrobial susceptibility patterns of elizabethkingia species. J Clin Med. (2020) 9:1431. doi: 10.3390/jcm9051431

23. Liu Y, Yang Y, Zhang C, Huang F, Wang F, Yuan J, et al. Clinical and biochemical indexes from 2019-nCoV infected patients linked to viral loads and lung injury. Sci China Life Sci. (2020) 63:364-74. doi: 10.1007/s11427-020-1643-8

24. Xie T, Wang Y, Fu S, Wang W, Xie C, Zhang Y, et al. Predictors for intravenous immunoglobulin resistance and coronary artery lesions in Kawasaki disease. Pediatr Rheumatol Online J. (2017) 15:17. doi: 10.1186/s12969-017-0149-1

25. Wyczalkowska-Tomasik A, Czarkowska-Paczek B, Zielenkiewicz M, Paczek L. Inflammatory markers change with age, but do not fall beyond reported normal ranges. Arch Immunol Ther Exp. (2016) 64:249-54. doi: 10.1007/s00005-015-0357-7

26. Michaud M, Balardy L, Moulis G, Gaudin C, Peyrot C, Vellas B, et al. Proinflammatory cytokines, aging, and age-related diseases. J Am Med Dir Assoc. (2013) 14:877-82. doi: 10.1016/j.jamda.2013.05.009

27. Veering BT, Burm AG, Souverijn JH, Serree JM, Spierdijk J. The effect of age on serum concentrations of albumin and alpha 1-acid glycoprotein. Br J Clin Pharmacol. (1990) 29:201-6. doi: 10.1111/j.1365-2125.1990.tb0 3620.x

28. Salive ME, Cornoni-Huntley J, Phillips CL, Guralnik JM, Cohen HJ, Ostfeld AM, et al. Serum albumin in older persons: relationship with age and health status. J Clin Epidemiol. (1992) 45:213-21. doi: 10.1016/0895-4356(92)90081-W

29. Lu J, Huang Y, Wang Y, Li Y, Zhang Y, Wu J, et al. Profiling plasma peptides for the identification of potential ageing biomarkers in Chinese Han adults. PLoS ONE. (2012) 7:e39726. doi: 10.1371/journal.pone.00 39726

30. Han K, Wang S, Jia W, Cao W, Liu M, Yang S, et al. Serum albumin and activities of daily living in Chinese centenarians: a cross-sectional study. BMC Geriatr. (2020) 20:228. doi: 10.1186/s12877-020-01631-7

31. Fulks M, Stout RL, Dolan VF. Serum globulin predicts all-cause mortality for life insurance applicants. J Insur Med. (2014) 44:93-8.

Conflict of Interest: The authors declare that the research was conducted in the absence of any commercial or financial relationships that could be construed as a potential conflict of interest.

Copyright (C) 2020 Feketea and Vlacha. This is an open-access article distributed under the terms of the Creative Commons Attribution License (CC BY). The use, distribution or reproduction in other forums is permitted, provided the original author(s) and the copyright owner(s) are credited and that the original publication in this journal is cited, in accordance with accepted academic practice. No use, distribution or reproduction is permitted which does not comply with these terms. 